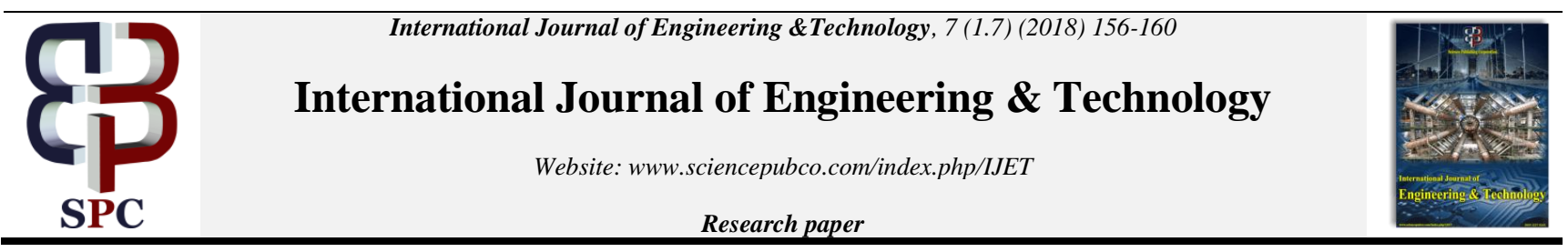

\title{
A swift unrest horde system for curtail SDO hit in cloud computing
}

\author{
S Ravikumar', E Kannan² \\ ${ }^{I}$ Assistant Professor, Dept of CSE, Vel Tech Rangarajan Dr.Sagunthala R\&D Institute of Science and Technology \\ ${ }^{2}$ Professor, Dept of CSE, Vel Tech Rangarajan Dr.Sagunthala R\&D Institute of Science and Technology \\ *Corresponding author E-mail: ravikumars.086@gmail.com
}

\begin{abstract}
One of the immense risk to benefit accessibility in distributed computing is Distributed Denial of Service. Here a novel approach has been proposed to limit SDO [Strewn Defiance of Overhaul] assaults. This has been wanted to accomplish by a canny quick motion horde organize. An astute horde arrange is required to guarantee independent coordination and portion of horde hubs to play out its handing-off tasks. Clever Water Drop calculation has been adjusted for appropriated and parallel advancement. The quick motion system was utilized to keep up availability between horde hubs, customers, and servers. We have intended to reproduce this as programming comprising of different customer hubs and horde hubs
\end{abstract}

Keywords: Horde, Cloud Computing, SDO.

\section{Introduction}

Distributed denial of service (SDO) causes execution of a site or cloud administration to be extremely debased. Utilizing SDO assaults, aggressors can control the flexibility of data by making certain data accessible at specific circumstances to figure out what data is and isn't transferred to open. As per a current overview, SDO assaults are seen as the main security danger. Fighting SDO additionally requires the most assets. The multiplication of SDO applications, for example, Low Orbit Ion Cannon (LOIC) [1] by 4 Chan-partnered programmers enables anybody to arrange and dispatch SDO assaults on any objective. As of late expert Wikileaks activists utilized LOIC to incredible impact.

Our article proposes utilizing a horde system to expand the serviceability of cloud benefits under SDO assaults. In case of an approaching SDO assault on a cloud benefit, the system will recompose itself to give the most noteworthy accessible administration level for the customers and servers under unfriendly system conditions.

Numerous arrangements have been proposed [2-9]; be that as it may, they have met with restricted achievement. We influence utilization of a horde to network to facilitate and hand-off messages amongst customers and servers. This horde framework can self-arrange and direct huge scale parallel look for ideal transfer arrangements. We have additionally investigated how horde insight can be consolidated into the current Internet foundation. Utilizing set up correspondence conventions, we have diminished the requirement for alterations of existing systems for simple selection. This approach exhibits the principal endeavor at making non-stationary horde transport for cloud administrations

\section{Related Works}

\subsection{Malevolent Traffic Detection and Rate Limiting}

Pushback [5] utilizes two procedures: total clog control (ACC) and pushback. Neighborhood ACC distinguishes clog at the switch level and devises an assault/blockage signature that can be converted into a switch channel. The mark characterizes a high data transfer capacity total, a subset of system activity, and nearby ACC decides a proper rate constrain for this total. Pushback spreads this rate confine for the total to the transitional upstream neighbors that contribute the biggest measure of the activity. This system works best against flooding-based assaults as they are dealt with as blockage wonders.

D- Locale intends to recognize assaults as they leave the system. It accumulates two-way movement measurements from the external outskirt at the source system and thinks about them to arrange activity models, reflecting typical/authentic, transient (suspicious) and assault conduct. In view of these models, DLOCALE applies rate limits at the switch on all the active switches, backing off the assault associations. DLOCALE can distinguish assaults; in any case, it stops assaults at source systems, and subsequently requires across the board sending to accomplish the coveted adequacy.

\subsection{Overlay Networks and Indirection Infrastructures}

SOS [4] with the objective of steering just great movement to servers. Just activity from great clients/customers is permitted. Customers influence utilization of an overlay to network to achieve the servers. The implicit excess of SOS, and also the mystery of how bundles are sent in the system, adds to its protection against SDO assaults. Be that as it may, this is a simply target-side arrangement, which can in any case be conceivably overpowered by a data transfer capacity assault at its entrance focuses

A spread-range like correspondence demonstrate. They proposed spreading their bundles haphazardly over all entrance focuses. Close by these parcels, a token is utilized for verification. The 
framework means to keep the assailant from assaulting particular overlay hubs by listening in on customers.

C. Saranya Jothi et.al [8] propose a multipurpose Internet Indirection Infrastructure (I3) by giving meeting based correspondence. Stoica et al. sets customer's information with an identifier. The servers make utilization of a trigger to show their enthusiasm for parcels. This foundation does not require the sender to know about the recipient and the other way around

Comprising of three fundamental parts. Phalanx depends on using a horde that can coordinate an assaulting botnet in quality. It makes utilization of basic parcel letter drops to enable clients to store and get messages. The servers in the system should expressly ask for a parcel from the letter drop for it to be conveyed. Finally, Phalanx utilizes verification components to guarantee that information put away in its system is honest to goodness. Be that as it may, Phalanx's message exchange component requires broad change of existing administrations.

\subsection{Augmentations to Server Applications}

It displays human conduct as a way to separate amongst human and bots. They recommend displaying demand elements of real clients. They utilize a specific server's history to prepare a discovery demonstrate. They predominately utilize the timings of solicitations as info. They additionally make utilization of the connections got to decide whether the customer is a human or a bot. They show prominence of the connections as a demand chart. This diagram is then coordinated to the entrance designs for the framework to separate bots. They have additionally proposed implanting imperceptible articles with hyperlinks. These items will just pull in the consideration of bots, and this is then used to signal the vindictive customers.

A bit expansion that gives multitiered validation and confirmation control. Slaughter Bots gives confirmation through graphical tests. At the point when bots overlook or can't settle the test, they are boycotted

\subsection{Approach and Strategy}

A powerful answer for expand serviceability of cloud benefits under SDO assaults is to actualize a decentralized horde transport framework. We can hand-off messages amongst customer and servers using the horde transport framework.

The horde organize is based on two fundamental ideas:

- Quick transition procedure in area name servers

- Association of horde

Quick motion facilitating uses an incredible number of hubs to give high accessibility to a specific asset. A quick motion benefit does not furnish an aggressor with a solitary IP address as an objective. The goal of any SDO assault is to center noxious movement from different sources to a bottleneck in a casualty's system. This bottleneck is regularly the focused on server's connect to the Internet. In a quick motion organize, there is no conspicuous bottleneck, in this manner making it harder for an assailant to cut down the whole administration arrange.

Quick transition facilitating permits a completely qualified space name to have numerous IP delivers doled out to it. It utilizes a brief timeframe to-live (TTL) parameter for a specific name record. The hostnames will be reassigned at high recurrence. A heap adjusting plan is for the most part set up to circulate the heap to speedier reacting has. It will likewise check the soundness of its hubs, and evacuates those that are inert. Utilizing quick transition, sites can keep up to a great degree high accessibility with numerous temperamental hosts. Figure 1 demonstrates how quick motion work.

In any case, a quick transition facilitating method in itself isn't sufficiently vigorous to adapt to modern SDO assaults that endeavor quick motion benefit systems. Assailants can basically train their SDO assaults to over-burden the known hosts of the system.

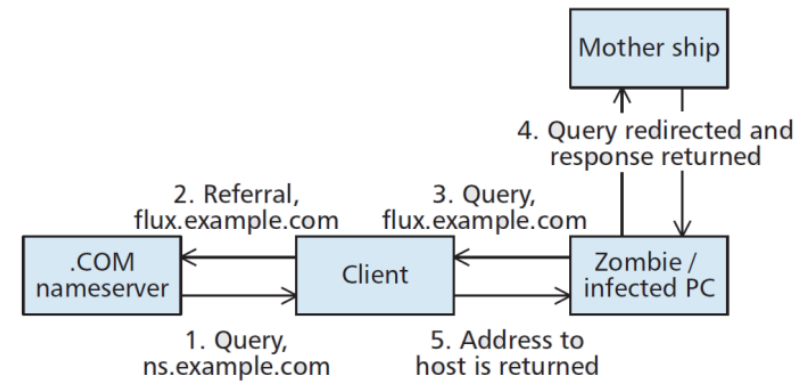

Figure 1. Task of a quick transition benefit organize.

This is on the grounds that quick motion systems give an alternate however static course to the assigned host. Despite the fact that associations are steered diversely whenever, there is no methods for it to redesign as the system condition changes. Consequently, by sniffing a quick transition organize, an aggressor will even now have the capacity to devise intends to disable the system by focusing on bottlenecks.

We apply methods related with horde insight to permeate the system with self-governance to address the above false notion. The self-arranging capacity of the horde organize gives straightforLocale mandates to it to improve its own particular system. This enables it to dependably give the most proficient handingoff of messages, notwithstanding when part of the system ends up unserviceable under an assault.

The development of waterways in nature motivates the Swift Water Drop (IWD) calculation [9]. Water dependably finds the easy way out. This is appropriate to outlining a hand-off framework for the horde arrange. The IWD calculation has two principle parameters: speed and soil. These two parameters change amid the lifetime of the IWD calculation. As the IWD moves from the source to the goal, it influences the dirt it goes through and may pick up or lose speed. As the IWD moves starting with one place then onto the next, its speed increments at a rate that is nonlinearly contrarily corresponding to the measure of soil between the two focuses. Subsequently, IWD traveling through less soil will assemble more speed. The IWD likewise conveys soil alongside it, and the sum is controlled when required for the IWD to move between areas. The development of soil between the hubs shapes the memory of the system. The IWD will incline toLocale picking ways of slightest protection (or soil). Henceforth, the IWD calculation can be viewed as an improvement arrangement.IWD can perform halfway streamlining relying upon the parameters it can detect. It is additionally exceptionally impervious to sudden system changes because of its disseminated nature. To deal with the huge number of horde hubs, a various leveled group approach is utilized. This is depicted further later. Most SDO moderation approaches require broad alteration of the system it wishes to ensure. This outcomes in a conservative obstruction to down to earth usage. Our horde goes about as a straightforLocale transport layer. It permits transmission of regular entrenched conventions (e.g., HTTP, SMTP) through its Network 


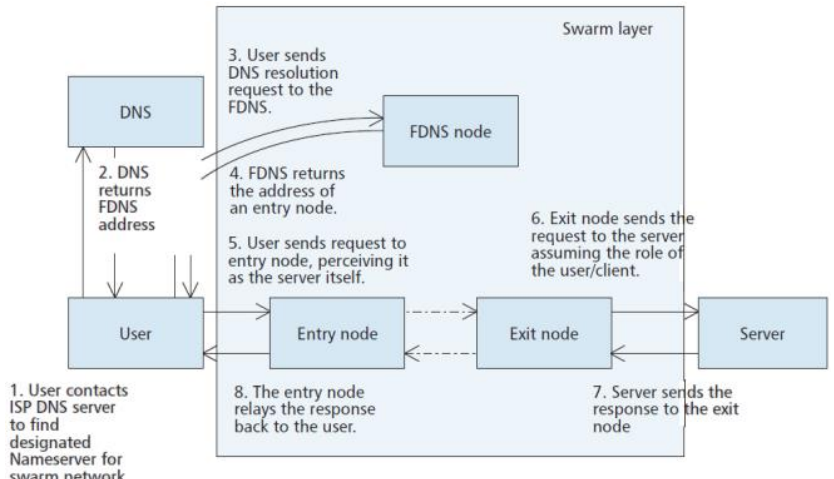

Figure 2. Task of transfer instrument.

No change on the server or customer side is required. Servers who need to profit by the horde arrange just need to enroll their IP locations and space names with the horde organize. The horde's name servers will course any demand to the predetermined area name to the server. Any reaction from the server will likewise be transferred without alteration to the customer. Applying our way to deal with existing administrations won't be discernable to the clients (the two servers and customers), and is subsequently effectively adoptable by cloud administrations.

\subsection{Hand-off Mechanism}

Traditional interchanges today forLocale solicitations and reactions through a stationary system. Utilizing quick motion methods we can build up a non-stationary and variable system framework. In ordinary correspondences, for example, customary serverclient engineering, demands from the customer are sent along the switches toLocales the server. The proposed system adds a layer to the stream of correspondences. Every approaching solicitation are enrolled at the horde, and the horde arranges and advances the solicitations to the assigned server. The server's reaction will be sent back to the horde, which will forLocale it to the asking for customer using session official. This procedure is delineated in Fig. 2. The hostnames will be reassigned at high recurrence.

The transition skilled area name server restores a progression of IP locations of its horde members. The IWD calculation decides the hubs transferred messages between the customer and server. The IWD regards the client and server as the begin and end hubs and the other way around. The name server just needs to restore the passage hub of a specific group. The people group of hubs would have independently contained nearby answers for the hand-off issue. The transition proficient area name server likewise goes about as a pioneer hub that remaining parts in consistent contact with the different horde groups.

\subsection{System Building}

Quick unresting gives an advantageous technique to horde building. As a hub ends up dynamic, it plays out a question on its assigned space name. In the event that no hubs are discovered, the hub accept an initiative or seed part. In the event that a hub can contact another hub when settling the assigned name, it will arrange and buy in to that specific group. Through performing dormancy minds neighboring hubs, every hub will decide whether it is in its ideal group. The ideal group is resolved from the accumulation of hubs that gives the most noteworthy throughput in its present arrangement. Different people group are shaped when a group has achieved a discretionary most extreme top. At the point when this top has been achieved, the group is partitioned in two; and from every group, the hub with the most noteworthy system execution will be elevated to the influential position. Groups exchange organize profile data through their pioneer hubs. This data incorporates the quantity of hubs and its locations. Pioneer hubs are then in charge of overseeing and spreading this data to its adherents. This procedure is outlined in Fig. 3.

Hybrids are urged to guarantee that every individual group stays lively. Companion data will be dispersed to every hub from the pioneer hub. Idleness tests will then be led between the hubs to decide appropriate breakaways. By enabling a gathering of hubs to traverse, we can find better arrangements. This procedure is motivated by hereditary calculations, whereby the choice to part from the group depends on a wellness work.

To decide the system landscape of the horde, we accept the system as a chart. By performing inertness test between hubs, the hubs will shape their own chart of their environment. Intermittently, every hub will impart its own insight to different hubs to enable this data to intertwine to frame a more extensive perspective of the system.

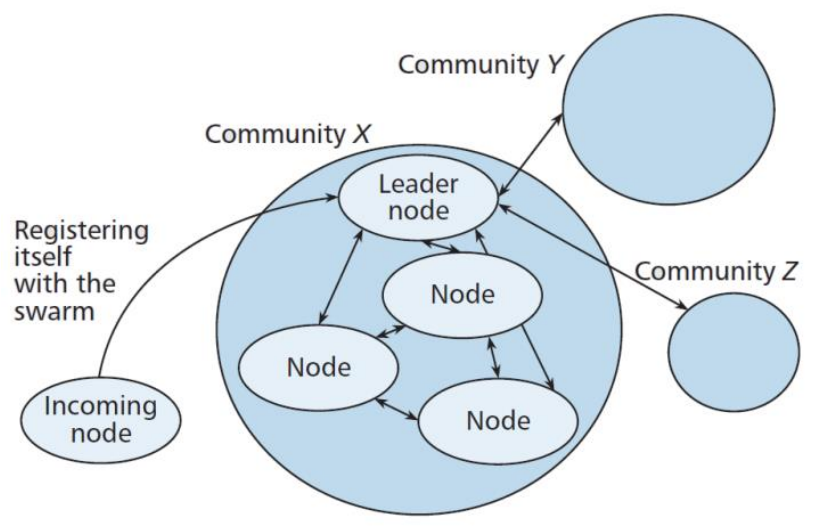

Figure 3. Horde communities.

This system landscape diagram is utilized as the reason for figuring the dirt parameter of every specific hub.

\subsection{Horde Directive}

The horde is comprised of comparative hubs dispersed in the system. The hubs speak with each other, performing decentralized coordination between them.

The horde makes utilization of the IWD procedure [10] to decide the quickest and most productive course to hand-off messages between the customer and servers. The speed and inactivity of the hubs are utilized as highlights for the IWD calculation. The measure of postponement brought about by experiencing a specific hub can be utilized as a measure of the measure of soil between two hubs.

The connected IWD calculation can be portrayed as takes after:

1. Introduction of the parameters. The diagram of the neighborhood group is instated in every hub. The nature of the globalbest arrangement is set to a self-assertive substantial negative number. The quantity of water drops is set to the quantity of hubs in the group. Each hub has a gone by hub list Vc(IWD), which is at first vacant. The speed of the IWD is set to an underlying quality.

2. As every one of the hubs are circulated into different groups, each will clutch a fractional answer for the worldwide streamlining issue. At every hub I, it will pick the following hub $\mathrm{j}$, inside the imperatives of the issue and not in the went to hub list with the accompanying likelihood show.

$f(\operatorname{soil}(i, j))$

$p_{i}{ }^{\text {IWD }}(j)=$

$\sum \mathrm{f}(\operatorname{soil}(\mathrm{i}, \mathrm{k}))$ 
Where $f(\operatorname{soil}(i, j))=$

$$
\begin{aligned}
& \varepsilon_{\mathrm{s}}+\mathrm{g}(\operatorname{soil}(\mathrm{i}, \mathrm{j})) \\
& g(\operatorname{soil}(i, f))=\left\{\begin{array}{cc}
\operatorname{soil}(i, j) & \text { if } \min (\operatorname{soil}(i, l)) \geq 0 \\
& k \notin v c(I W D)
\end{array}\right. \\
& \operatorname{soil}(i, j)-\min (\operatorname{soil}(i, f)) \text { otherwise }
\end{aligned}
$$

$$
\mathrm{k} \notin \mathrm{vc}(\mathrm{IWD})
$$

3. For each IWD moving starting with one hub then onto the next, the speed is refreshed.

$$
\text { velocity }^{\mathrm{IWD}}(\mathrm{t}+1)=\text { velocity }^{\mathrm{IWD}}+\frac{\mathrm{a}_{\mathrm{v}}}{\mathrm{b}_{\mathrm{v}}+\mathrm{c}_{\mathrm{v}} \cdot \operatorname{soil}^{2}(\mathrm{i}, \mathrm{j})}
$$

$a v, b v$, and $c v$ are parameters that decide the consistency of the water bead.

4For each IWD that moves starting with one hub then onto the next, the measure of soil change is connected with the speed of the moving water drop. This connection can be changed in accordance with produce substitute answers for improvements.

\section{The soil parameter is then refreshed as needs be.}

6 . The ideal way is registered utilizing a quality capacity. The worldwide best arrangement is recovered from the pool of arrangements.

7. The dirt on the way of the ideal arrangement is then refreshed.

8. This calculation is rehashed as the framework works and upto-date ideal ways will be produced.

The merging property of the IWD was appeared in [10].In the over, the IWD calculation utilizes a horde of water drops to direct a huge parallel look for the ideal arrangements.

\section{Discourse}

\subsection{Correlations with Existing Work}

The framework instills horde insight into a vehicle organize in a way like an indirection foundation. Our framework mitigates SDO by giving a versatile and powerful transport benefit. It doesn't unequivocally identify noxious movement as do Pushback [5] and D-LOCALE. Malevolent movement discovery and rate restricting requires specialist organizations' dedication and collaboration. This appearances monetary hindrances as specialist organizations sometimes see ensuring contenders' systems as being in their own particular self-intrigue. These instruments likewise require switches in the systems to be adjusted to help such highlights.

Methodologies, for example, demonstrating human conduct [7] and killbots [3] broaden the ability of existing server applications to alleviate SDO assault. However these methodologies does not address asset depletion assaults on a solitary target - Server. Improvement and discovery of bots lessens the viability of a SDO assault. Be that as it may, it does not have the capacity to adjust cautious system arrangements to diminish the effect of such the assaults on their administration level. Propelled by the developing pattern of decentralized shared frameworks, appropriated olutions, for example, overlay systems and indirection foundation have extraordinary potential as answers for the SDO chal-
lenge.Secure Overlay Services [4] gives a type of virtual system to encourage message going in a protected domain. Particles [9] as an advancement of overlay arranges additionally make utilization of stationary safe hubs to perform message passing.

Our horde-based approach speaks to a novel endeavor to make a non-stationary transport arrange. In correlation, the static idea of existing overlay systems keeps the system from reconfiguring itself when looked with regularly changing system territory. This firmness keeps existing arrangements from abusing the capability of extensive conveyed frameworks. Such overlay systems accept responsibility for overlay hubs. This provisioning of devoted equipment assets may be fetched restrictive for any single gathering. The horde system can be sent on numerous stages. They can likewise be woven into existing Internet applications to encourage the benefit of everyone. This is influenced conceivable by the vigorous idea of the horde to arrange. The quick transition abilities don't require any specific host to be serviceable at all conditions. This reductions the reliance on committed equipment for its proceeded with task.

\section{Impediments}

The proposed engineering is intended for stateless parcel transfer through a non-stationary system. The horde organize ties every customer to a server however a one of a kind course that exists just for a specific association. Each message will pass however a special course that isn't ensured for the following transmission. The message passing administration can be seen as a one-timeutilize just excursion. The course changes as indicated by the differing system territory. The customer will see the section hub as the server. The server expect that the leave hub of the horde as the asking for customer. This system keeps an immediate host-tohave association. While this gives the area obscurity that builds the security for the server, it doesn't permit certain associations, for example, SSL to be conceivable. Future work will deliver this worry to permit virtual industrious and secure association with occur.

It is vital to take note of that there are numerous variables that add to the overhead required keep up a horde arrange. TTL estimations of regular space names keep going for a drawn out stretch of time. TTLs for quick motion systems are short and furthermore inclined to disturbances. Contingent upon the unpredictability of the system, quick changes in the horde creation may bring about idle name servers recorded. This issue can be tended to by having a substantial sending of name servers to enhance heartiness. Be that as it may, expanding the assignment of name servers will bring about a deplete on the horde's assets.

Correspondence between hubs takes up the horde's data transfer capacity. The hubs habitually get in touch with each other for idleness tests and exchanging of associate data. While these messages are restricted to a level of accessible transfer speed, they speak to an overhead required to keep up the horde uprightness.

\section{Conclusion}

We have introduced a successful SDO moderation procedure comprising of hubs in a horde organize. The customer tries to achieve the server through a completely qualified area name. The customer is coordinated to the server and afterLocale sends its demand to Figure 5. System without insight under SDO assault. The assigned server by means of the group's leave hub. The server then reactions as needs be, and the outcome is sent through the horde organize back to the customer. The horde arrange is influenced available through quick to transition facilitating, thus showing high power. The horde organize continually reconfigures itself using a parallel improvement calculation, for example, the Swift Water Drop system. Every one of these methods are utilized to broaden the serviceability of a cloud benefit under 
SDO assault.To demonstrate the feasibility of such a horde network, a PC recreation utilizing an elite processing group was actualized. Up to 400,000 customers are utilized to associate with the 10,000-hub horde organize. Reenacted SDO assaults from 10,000 devoted assault hubs are led against the horde organize. Results have demonstrated that harms maintained by SDO assaults are enormously decreased with the utilization of a horde transport layer.

\section{References}

[1] R. Geetha and E. Kannan, “A Novel Resource Constraint Secure(Rcs) Routing Protocol For Wireless Sensor Network" in Journal of Engineering Science and Technology,Vol. 12, No. 2 (2017) $518-529$

[2] C. Dixon, T. Anderson, and A. Krishnamurthy, "Phalanx: Withstanding Multimillion-Node Botnets," Proc. 5th USENIX Symp. Networked Systems Design and Implementation, San Francisco, CA, 2008.

[3] S. Kandula et al., "Botz-4-Sale: Surviving Organized SDO Attacks That Mimic Flash Crowds," Proc. 2nd Symp. Networked Systems Design and Implementation, 2005.

[4] A. D. Keromytis, V. Misra, and D. Rubenstein, "SOS: Secure Overlay Services,"SIGCOMM Comp. Commun. Rev., vol. 32, 2002, pp. 61-72.

[5] R. Mahajan et al., "Controlling High Bandwidth Aggregates in the Network,"SIGCOMM Comp. Commun. Rev., vol. 32, 2002, pp. 62-73.

[6] M. J. Carmel Mary Belinda and E. Kannan , "Straight Line Routing on Next Hop Graph in Wireless Sensor Networks" in Journal of Computer Science

[7] S.Ravikumar, M.Viswanathan and G.S.Raj, "A Dossier Fortification With Cover-Up And Load Balanced Structure" in International Journal of Applied Engineering Research Volume 10, Number 7 (2015) pp. 18469-18476 @ Research India Publications.

[8] C. Saranya Jothi, Ravikumar S, Antony Kumar K and A.Suresh , "An Approach for verifying correctness of web service compositions" in International Journal of Engineering \&Technology, 7 (1.7) (2018) 5-10

[9] Antony Kumar K, C Saranya Jothi, S Ravikumar, and V Usha, "Wireless body area network using data communication protocol" in International Journal of Engineering \&Technology, 7 (1.7) (2018) 112-114 